\title{
How image-processing parameters can influence the assessment of dental materials using micro-CT
}

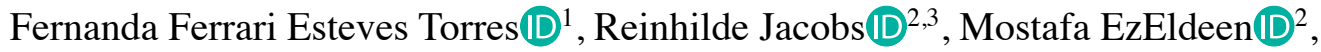 \\ Karla de Faria-Vasconcelos $\mathbb{D}^{2}$, Juliane Maria Guerreiro-Tanomaru(Dי1 ${ }^{1}$, \\ Bernardo Camargo dos Santos $\mathbb{D}^{4}$, Mário Tanomaru-Filho $\mathbb{D}^{1, *}$ \\ ${ }^{1}$ Department of Restorative Dentistry, São Paulo State University (UNESP), School of Dentistry, Araraquara, SP, Brazil \\ ${ }^{2}$ OMFS IMPATH Research Group, Department of Imaging and Pathology, Faculty of Medicine, KU Leuven and Oral and Maxillofacial \\ Surgery, University Hospitals Leuven, Leuven, Belgium \\ ${ }^{3}$ Department of Dental Medicine, Karolinska Institute, Stockholm, Sweden \\ ${ }^{4}$ Department of Nuclear Energy, Federal University of Rio de Janeiro (UFRJ), Rio de Janeiro, RJ, Brazil
}

\section{ABSTRACT}

\begin{abstract}
Purpose: The aim of this study was to evaluate the influence of voxel size and different post-processing algorithms on the analysis of dental materials using micro-computed tomography (micro-CT).

Materials and Methods: Root-end cavities were prepared in extracted maxillary premolars, filled with mineral trioxide aggregate (MTA), Biodentine, and Intermediate Restorative Material (IRM), and scanned using micro$\mathrm{CT}$. The volume and porosity of materials were evaluated and compared using voxel sizes of 5, 10, and $20 \mu \mathrm{m}$, as well as different software tools (post-processing algorithms). The CTAn or MeVisLab/Materialise 3-matic software package was used to perform volume and morphological analyses, and the CTAn or MeVisLab/Amira software was used to evaluate porosity. Data were analyzed using 1-way ANOVA and the Tukey test $(P<0.05)$.

Results: Using MeVisLab/Materialise 3-matic, a consistent tendency was observed for volume to increase at larger voxel sizes. CTAn showed higher volumes for MTA and IRM at $20 \mu \mathrm{m}$. Using CTAn, porosity values decreased as voxel size increased, with statistically significant differences for all materials. MeVisLab/Amira showed a difference for MTA and IRM at $5 \mu \mathrm{m}$, and for Biodentine at $20 \mu \mathrm{m}$. Significant differences in volume and porosity were observed in all software packages for Biodentine across all voxel sizes.

Conclusion: Some differences in volume and porosity were found according to voxel size, image-processing software, and the radiopacity of the material. Consistent protocols are needed for research evaluating dental materials. (Imaging Sci Dent 2020; 50: 161-8)
\end{abstract}

KEY WORDS: Dental Materials; Image Processing, Computer-Assisted; Imaging, Three-Dimensional; X-ray Microtomography

\section{Introduction}

Dental research has revealed the importance of 3-dimensional (3D) imaging in the clinical field and in research-

This work was supported in part by the Coordenação de Aperfeiçoamento de Pessoal de Nível Superior, Brasil (CAPES) Finance Code 001, and was fully supported by the São Paulo Research Foundation - FAPESP (grant numbers 2016/00321-0, 2017/22481-1, and 2017/19049-0).

Received November 19, 2019; Revised February 14, 2020; Accepted February 20, 2020 *Correspondence to : Prof. Mário Tanomaru-Filho

Department of Restorative Dentistry, Araraquara Dental School, São Paulo State University - UNESP, Rua Humaitá, 1680, CEP 14801-903 Araraquara, SP, Brazil Tel) 55-16-3301-6390, E-mail) tanomaru@uol.com.br ing different materials. ${ }^{1}$ Micro-computed tomography (micro-CT) is a 3D, non-destructive imaging method that is considered the gold standard for in vitro applications, ${ }^{2}$ since it provides more detailed information than other imaging modalities about small structures. ${ }^{3}$ Micro-CT has often been used to assess cement volume $\mathrm{e}^{4,5}$ and to determine obturation porosity. ${ }^{6,7}$ However, the imaging-based evaluation of dental materials still poses a challenge in some cases, since the currently available cements can generate artifacts, which decrease overall image quality. ${ }^{2}$ Moreover, there is a lack of standardization of image-processing pa-

Copyright (c) 2020 by Korean Academy of Oral and Maxillofacial Radiology

This is an Open Access article distributed under the terms of the Creative Commons Attribution Non-Commercial License (http://creativecommons.org/licenses/by-nc/3.0) which permits unrestricted non-commercial use, distribution, and reproduction in any medium, provided the original work is properly cited. Imaging Science in Dentistry · pISSN 2233-7822 eISSN 2233-7830 
rameters, which can influence the results of research.

Segmentation of the region of interest influences the accuracy of analyses of micro-CT images. ${ }^{8}$ Several segmentation methods are available and may have distinct influences on the outcomes. ${ }^{9}$ Manual segmentation is a time-consuming task requiring an experienced operator, with low repeatability and reproducibility. ${ }^{10}$ Conversely, semi-automatic and automatic methods are faster, and new, less operator-dependent mathematical processing algorithms are being developed to process large volumes of data. ${ }^{11}$

Image analysis is a fundamental part of research after the image segmentation step. ${ }^{12}$ Morphometric indices can be derived from either a simple voxel-counting method or more advanced volume-rendering methods. Various manufacturers provide different software packages that can be used to compute these indices. ${ }^{13}$

The demands placed on image segmentation and analysis become even more stringent when the image quality is limited due to low spatial resolution and contrast, or a large amount of noise. ${ }^{14}$ In this challenging situation, improved image resolution may assist the computer (algorithm) to better define the boundaries of regions and structures. ${ }^{11,15-17}$ Although high resolution provides some advantages, such as greater precision, ${ }^{18}$ the drawback is that it requires a greater amount of data, a longer scanning time, and more intensive analysis, ${ }^{19}$ resulting in higher costs and longer rendering times.

Imaging processing parameters for evaluating the volume and porosity of dental materials using micro-CT have still not been standardized. Consequently, researchers have not been able to assess the differences observed in imaging investigations using different spatial resolutions or software to perform the analyses. Recommendations for imaging protocols are needed to reduce measurement error and scanning time. ${ }^{20}$ Thus, the aim of this study was to evaluate the impact of voxel size and different post-processing algorithms on the precision of measurements of the volume and porosity of dental materials.

\section{Materials and Methods}

\section{Specimen preparation}

After obtaining approval from the Institutional Ethics Committee (Registration number \#CAAE: 9779617.5.0000. 5416), 12 extracted 2-rooted maxillary premolars without any anomalies in the root canal system were selected. Root-end resection was performed at $90^{\circ}$ from the long axis of the root, approximately $3 \mathrm{~mm}$ from the apex. After apicectomy, 3.0-mm deep cavities were prepared using an Ultrawave device (Ultrawave XS, Ultradent, South Jordan, UT, USA) with a T1F-R ultrasonic retrotip (CVD-Vale, São José dos Campos, SP, Brazil).

The teeth were divided randomly into 3 groups, and the cavities were filled with mineral trioxide aggregate (MTA) (Angelus, Londrina, PR, Brazil), Biodentine (Septodont, Saint Maur des Fosses, France) or Intermediate Restorative Material (IRM) (Dentsply DeTrey, Konstanz, Germany) by a single, previously trained and calibrated operator. The materials were mixed according to the respective manufacturer's instructions. The samples were kept in an oven at $37^{\circ} \mathrm{C}$ and $95 \%$ humidity for 3 times longer than their recommended setting time.

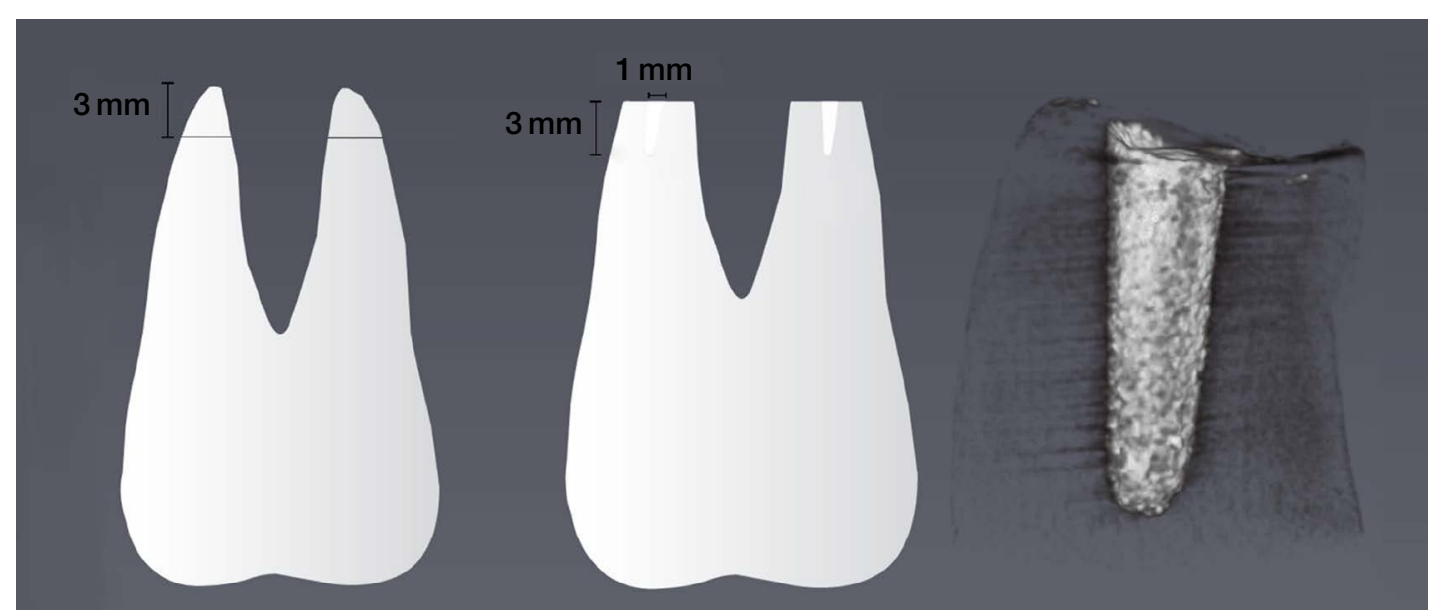

Fig. 1. Schematic figure represents tooth preparation and evaluation of the materials. Root-end resection was performed at $90^{\circ}$ from the long axis of the root, approximately $3 \mathrm{~mm}$ from the apex. Thus, 3.0-mm-deep cavities were prepared and filled with the root-end filling materials. The materials were assessed after micro-computed tomography scanning and image reconstruction. 


\section{Image acquisition and reconstruction}

The specimens were scanned using micro-CT (SkyScan 1272, Bruker, Kontich, Belgium) with the following acquisition parameters: $5 \mu \mathrm{m}$ voxel size, $100 \mathrm{kVp}, 100 \mu \mathrm{A}$, $0.11 \mathrm{~mm}$ copper filter, 4 frame average, and $180^{\circ}$ rotation. The datasets were reconstructed using NRecon software (v1.6.10.4; Bruker, Kontich, Belgium). The correction parameters were $40 \%$ for smoothing, $25 \%$ for beam hardening, and $25 \%$ for ring artifacts. All the datasets were exported using the Digital Imaging and Communications in Medicine file format with an isotropic voxel size of $5 \mu \mathrm{m}$. A schematic illustration of specimen preparation and evaluation is presented in Figure 1.

\section{Image segmentation and volume calculation}

The filling materials were segmented and their total volume was recorded at 5, 10, and $20 \mu \mathrm{m}$. Segmentation was performed using 2 different protocols. The CTAn (V1.15.4.0; Bruker, Kontich, Belgium) protocol uses adaptive thresholding, bearing in mind that segmentation can be improved by using local threshold values instead of a single global threshold value. ${ }^{21}$ In this method, the examiner segments the images visually, and records the threshold values for each sample. ${ }^{9}$ The MeVisLab (MeVis Research, Bremen, Germany) protocol uses a semi-interactive livewire boundary extraction, ${ }^{10}$ in which the user moves the mouse from a manually selected point, and a livewire boundary selects and wraps around the object of interest. New points are then selected, until the boundary has been delimited and the area has been segmented. ${ }^{22}$ This method creates a set of orthogonal contours, and then applies a variational interpolation algorithm that reconstructs the surface of the object, ${ }^{23,24}$ providing an interactive segmentation that combines examiner recognition to outperform the ability of the computer itself to achieve synergistic delineation. ${ }^{22}$

After segmentation using MeVisLab, the reconstructed 3D surfaces were exported to 3-matic (Materialise; Leuven, Belgium) for volume quantification and morphological analysis by superimposition of the reconstructed 3D surfaces $(5,10$, and $20 \mu \mathrm{m})$ to obtain a color-coded map showing the surface deviation of the segmented objects.

\section{Porosity analysis of the materials}

After segmentation by CTAn, the porosity of each material was calculated using the same package. In comparison, after the material was segmented using MeVisLab, the image was exported to Amira (Amira, FEI Visualization Sciences Group, Merignac, France) to calculate porosity. The pores were segmented using top-hat transformation.

\section{Statistical analysis}

All data were analyzed with the GraphPad Prism 7.00 statistical software package (GraphPad Software, La Jolla, CA, USA). One-way analysis of variance was used to evaluate the statistical significance of differences according to voxel size, as well as differences in the volume and porosity measurements using various software packages at 5,10 , and $20 \mu \mathrm{m}$. The level of significance was set at $P<0.05$.

\section{Results}

\section{Volume of the materials}

Table 1 presents the volume measurements. MTA and IRM showed significantly higher volume results only when

Table 1. Volume and porosity values using different voxel sizes and software packages to analyze the dental materials

\begin{tabular}{|c|c|c|c|c|c|}
\hline & & \multicolumn{2}{|c|}{ Volume $\left(\mathrm{mm}^{3}\right)$} & \multicolumn{2}{|c|}{ Porosity (\%) } \\
\hline & & MeVisLab/Materialise 3-matic & CTAn & MeVisLab/Amira & CTAn \\
\hline \multirow[t]{3}{*}{ MTA } & $5 \mu \mathrm{m}$ & $1.24 \pm 0.19 *$ & $1.23 \pm 0.19$ & $10.12 \pm 2.57 *$ & $9.11 \pm 1.26^{*}$ \\
\hline & $10 \mu \mathrm{m}$ & $1.28 \pm 0.20 *$ & $1.24 \pm 0.19$ & $8.17 \pm 1.92$ & $8.53 \pm 1.41 *$ \\
\hline & $20 \mu \mathrm{m}$ & $1.39 \pm 0.21 *$ & $1.25 \pm 0.19 * \dagger$ & $7.78 \pm 1.75$ & $6.93 \pm 1.71^{*}$ \\
\hline \multirow[t]{3}{*}{ Biodentine } & $5 \mu \mathrm{m}$ & $1.24 \pm 0.11 *$ & $1.13 \pm 0.11^{* \dagger}$ & $6.24 \pm 1.00$ & $4.95 \pm 1.22 * \dagger$ \\
\hline & $10 \mu \mathrm{m}$ & $1.30 \pm 0.09 *$ & $1.14 \pm 0.11^{* \dagger}$ & $6.15 \pm 1.10$ & $4.40 \pm 1.17 * \dagger$ \\
\hline & $20 \mu \mathrm{m}$ & $1.38 \pm 0.12 *$ & $1.15 \pm 0.11^{* \dagger}$ & $5.17 \pm 0.95^{*}$ & $3.19 \pm 1.02 * \dagger$ \\
\hline \multirow[t]{3}{*}{ IRM } & $5 \mu \mathrm{m}$ & $1.18 \pm 0.23^{*}$ & $1.18 \pm 0.12$ & $8.13 \pm 1.25 *$ & $7.77 \pm 1.29 *$ \\
\hline & $10 \mu \mathrm{m}$ & $1.23 \pm 0.23^{*}$ & $1.20 \pm 0.17$ & $6.81 \pm 1.75$ & $7.37 \pm 1.31 *$ \\
\hline & $20 \mu \mathrm{m}$ & $1.37 \pm 0.23 *$ & $1.22 \pm 0.17 * \dagger$ & $5.34 \pm 1.00$ & $6.40 \pm 1.31 *$ \\
\hline
\end{tabular}

*: $P<0.05$ among the voxel sizes, $\uparrow: P<0.05$ between the software packages 


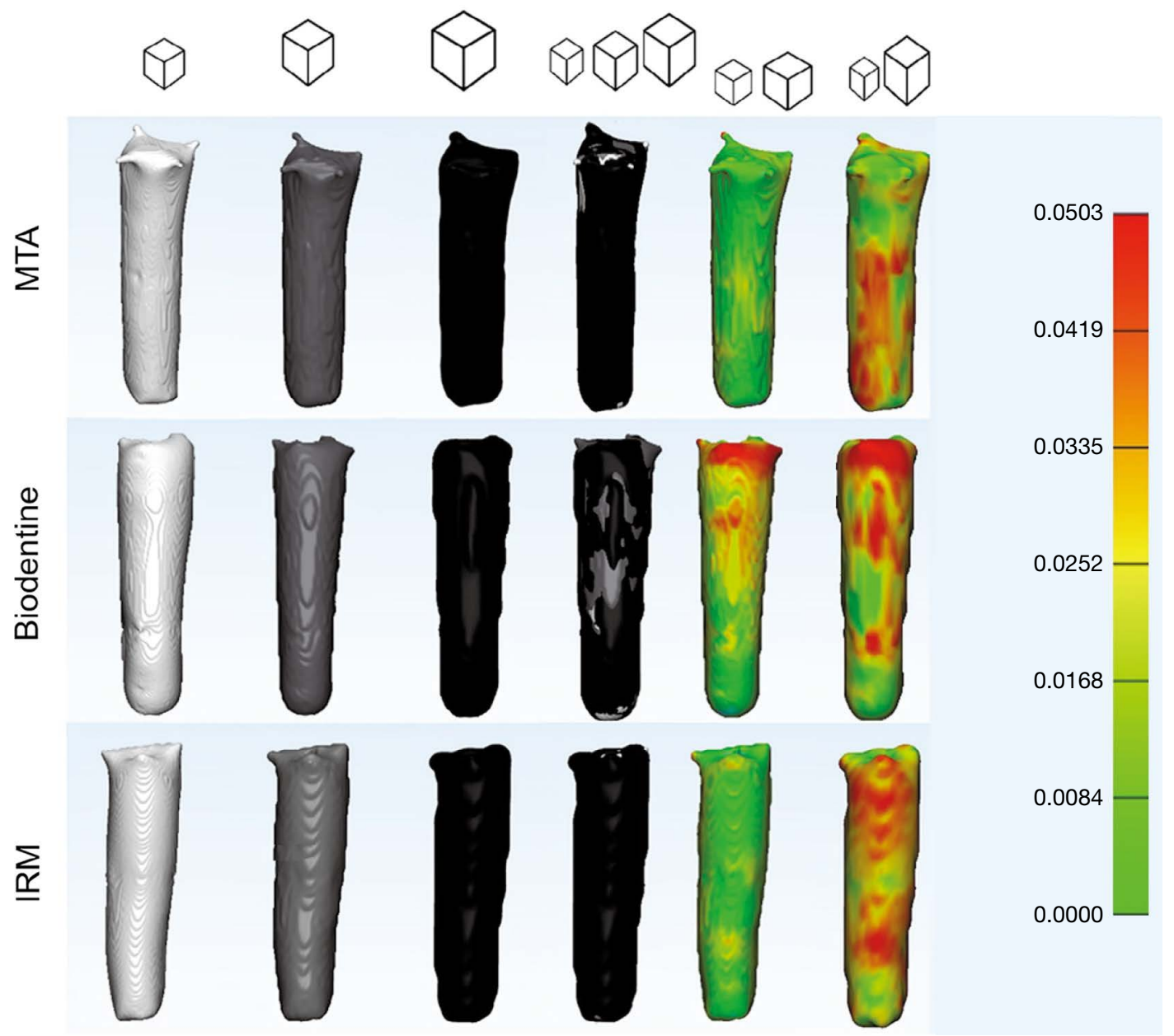

Fig. 2. Three-dimensional models of MTA, Biodentine, and IRM at $5 \mu \mathrm{m}$ (the small cube represents the smaller voxel size; white), $10 \mu \mathrm{m}$ (the medium cube represents the medium voxel size; gray), $20 \mu \mathrm{m}$ (the large cube represents the larger voxel size; black), and after superimposition between 5 and $10 \mu \mathrm{m}$ and between 5 and $20 \mu \mathrm{m}$ voxels. The color map shows the increase in volume (mm) after superimposition. MTA, mineral trioxide aggregate; IRM, Intermediate Restorative Material.

analyzed at $20 \mu \mathrm{m}$ by CTAn, whereas Biodentine had different results at all voxel sizes $(P<0.05)$. The same results were observed in the comparison between software packages; that is, the images for MTA and IRM were only significantly different at $20 \mu \mathrm{m}$, whereas the values obtained for Biodentine were different at all voxel sizes for each software program $(P<0.05)$. The increase in volume with increasing voxel size was statistically significant for all 3 cements when MeVisLab/Materialise 3-matic was used $(P<0.05)$ (Table 1). Figure 2 shows the 3D models of each material at 5,10 , and $20 \mu \mathrm{m}$, before and after superimposition using a color map that shows the increase in volume $(\mathrm{mm})$ when the voxel size was increased.

\section{Porosity of the materials}

The results for porosity, considering all voxel sizes and software packages, are presented in Table 1. Unlike vol- ume, the porosity of the materials decreased as voxel size increased (Table 1). Figure 3 shows 3D models representing the porosity of each material observed at 5,10 , and 20 $\mu \mathrm{m}$, with a color map that shows the thickness (mm) of the pores. According to CTAn, the porosity of MTA, IRM, and Biodentine showed significant differences according to voxel size $(P<0.05)$. According to the MeVisLab/Amira software, the porosity values for MTA and IRM were significantly different at $5 \mu \mathrm{m}(P<0.05)$, whereas the values at 10 and $20 \mu \mathrm{m}$ were similar $(P>0.05)$. Biodentine showed different results at $20 \mu \mathrm{m}(P<0.05)$.

The values obtained for MTA and IRM using CTAn and MeVisLab/Amira were similar $(P>0.05)$. In contrast, Biodentine showed significant differences according to the image analysis program used, independently of the voxel size $(P<0.05)$. 


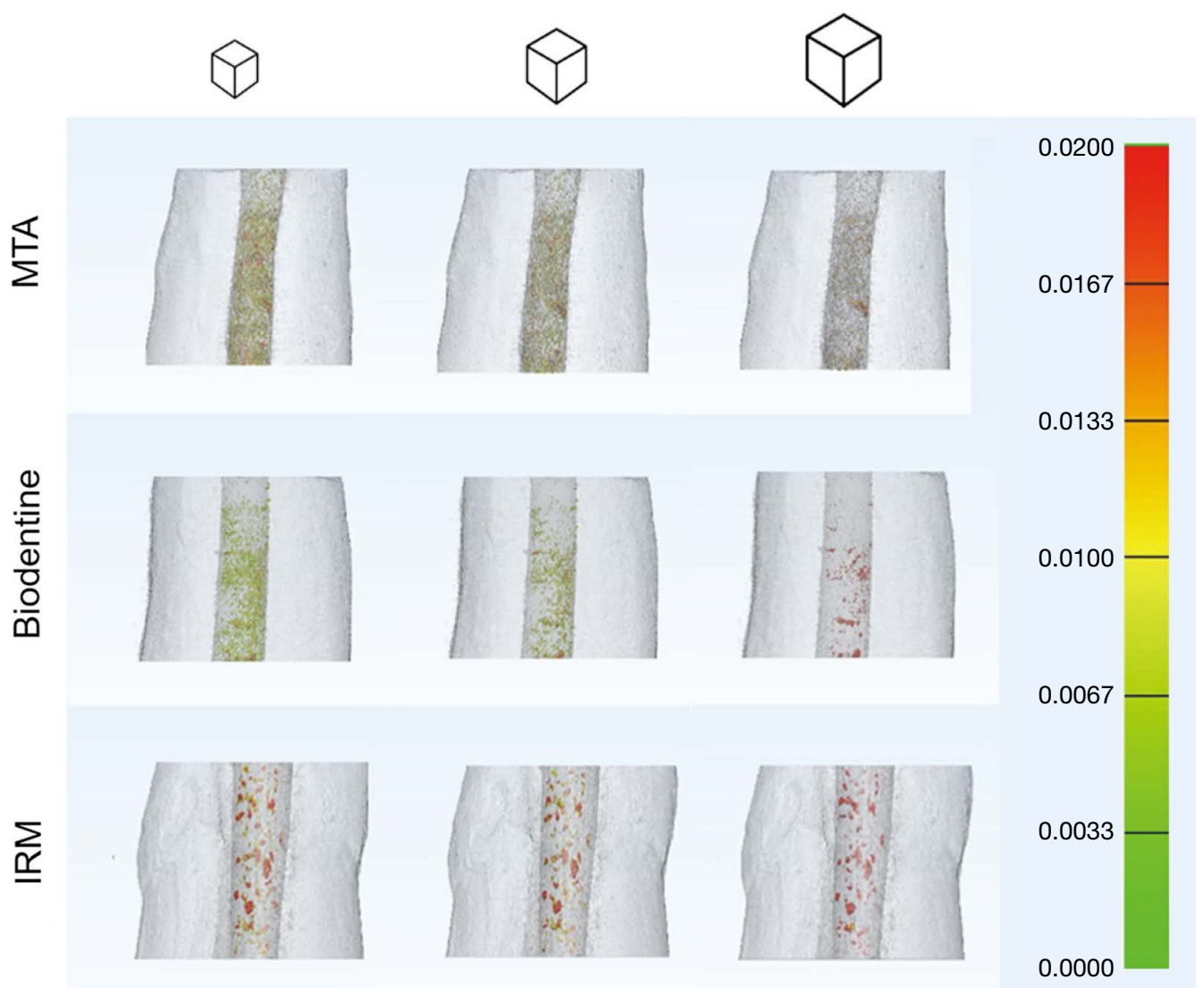

Fig. 3. Three-dimensional models represent the porosity of the MTA, Biodentine, and IRM cements at $5 \mu$ m (the small cube represents the smaller voxel size), $10 \mu \mathrm{m}$ (the medium cube represents the medium voxel size), and $20 \mu \mathrm{m}$ (the large cube represents the larger voxel size). The color map shows the thickness $(\mathrm{mm})$ of the pores.

\section{Discussion}

The evaluation of the volumetric and morphological stability and porosity of dental materials may play an important role in determining the most appropriate material for each treatment, thereby contributing to improvements in the clinical success rate over time. Many physicochemical properties of dental materials are poorly investigated, and the data published in the literature are controversial. ${ }^{25}$

In recent years, many innovative advances have occurred in high-resolution imaging modalities and in image post-processing procedures. ${ }^{26}$ High-resolution micro-CT imaging has been widely applied to evaluate dental materials using different approaches for image acquisition, image assessment, and outcome reporting. However, this has led to a lack of consistency that makes it difficult to interpret results and to compare findings across different studies. ${ }^{13}$ The current study shows that voxel size and post-processing algorithms influenced measurements of the volume and porosity of dental materials using micro-CT images.
Regarding the impact of voxel size on volumetric analyses of materials, increasing the voxel size from 5 to 10 $\mu \mathrm{m}$ resulted in increased volume, with an error of approximately $1 \%$ and $4 \%$ using CTAn and MeVisLab/Materialise 3-matic software, respectively. At $20 \mu \mathrm{m}$, the error increased to $2 \%$ using CTAn, and to $13 \%$ using MeVisLab/ Materialise 3-matic. Moreover, when Biodentine was used - as a material with low radiopacity ${ }^{27}$ - the differences according to voxel size and software package became more evident.

These findings can be attributed to the partial volume effect that occurs when neighboring voxels include multiple materials. During image acquisition, anatomical structures are distinguished by their radiographic density. Therefore, voxels at the external surface of the sample may contain the densities of 2 materials. ${ }^{28-30}$ Although parameters for image correction (smoothing, correction of beam hardening, and correction of ring artifacts) can be applied during the reconstruction of the images, the application of artifact reduction tools to micro-CT images might not have any 
significant influence on objective image analyses. ${ }^{31}$ Hence, lower spatial resolution associated with low contrast between structures ${ }^{32}$ may induce this kind of error, resulting in overestimation of some parameters, including thickness and volume. ${ }^{33}$

Conversely, porosity decreased as voxel size increased. A decrease of approximately $7 \%$ in porosity in response to increasing the voxel size from 5 to $10 \mu \mathrm{m}$ was observed using CTAn, and the corresponding decrease was $12 \%$ using MeVisLab/Amira. When images with $20-\mu \mathrm{m}$ voxels were evaluated, reductions in porosity of up to $26 \%$ and $24 \%$ were observed using CTAn and MeVisLab/Amira, respectively. This variation occurred because resolutions exceeding the dimensions of micropores, or very thin structures, preclude the visualization of small features. ${ }^{3,34}$

The fact that high porosity may be associated with a decrease in the flexural strength of dental materials ${ }^{6}$ makes the evaluation of this property clinically valuable. Accordingly, using a high resolution can help researchers better distinguish small pores and voids inside cements. ${ }^{35}$ In accordance with our results, other studies have demonstrated that cortical bone porosity measurements depend on voxel $\operatorname{size}^{16}$ and have suggested a voxel size of $11.2 \mu \mathrm{m}$ as a cutoff value for the evaluation of root canal filling voids. ${ }^{36}$

The differences observed in the current study highlight the influence of the post-processing algorithms on the analysis of dental materials by micro-CT. ${ }^{37}$ Thresholding can be performed by simple global methods or by adaptive methods using CTAn software. ${ }^{9}$ Our study used the adaptive method because it applies local threshold values instead of a single global method. As a result, segmentation could be improved by determining the optimal threshold value of each voxel within its neighborhood. ${ }^{21}$ Segmentation using MeVisLab was performed by applying interactive livewire boundary extraction to select the region of interest from the surrounding area. ${ }^{24}$ This is an interactive tool that provides efficient, accurate, and reproducible boundary extraction. ${ }^{10}$

Image analysis is the next most critical step after segmentation. Different algorithms have been developed to compute several indices. ${ }^{13}$ When performing a 3D morphometric analysis using CTAn, the marching cubes algorithm is used to calculate values for surfaces and volumes, ensuring more accurate measurements than simple pixel counting techniques. This algorithm is a traditional method preferred for its simplicity and efficiency. ${ }^{38}$ Volume analysis using Materialise 3-matic was performed by importing an STL file after segmentation of the dental materials using MeVisLab. Since STL files are surface representations, a tetrahedral volume was generated from the triangle sur- face mesh to perform the analysis in 3-matic. This method allows the user to determine what parameters and settings are most important, depending on the application and the user's preferences. The resulting algorithm produces an effective assessment. ${ }^{39}$

The segmented images acquired with MeVisLab were also used to perform porosity analysis by Amira. The black top-hat transform algorithm was applied in this software to perform pore segmentation. Total porosity was then calculated as a percentage of the overall volume. Black top-hat transformation is an image-processing technique for extracting dark features on a variable background that offers more accurate volume estimations. ${ }^{40}$

The smallest possible voxel size should preferably be used for scans of small structures in order to maximize the accuracy of the measurements..$^{18,21,41,42}$ However, higher-resolution scans require longer acquisition times, because they must collect more projections and generate large datasets. Therefore, the tradeoff between voxel size and scan time should be carefully considered. ${ }^{13}$ It is important to emphasize that the variations in the values obtained using different software packages were more meaningful when larger voxels were used. Nevertheless, although our results show that smaller voxel sizes are advantageous, the use of larger voxels should not be discouraged when appropriate.

In conclusion, a micro-CT analysis of the volume and porosity of dental materials showed distinct outcomes based on voxel size, the post-processing algorithm, and the radiopacity of the material. The similarities among the software-based outcomes and the reliability of the results increased with smaller voxel sizes and more radiopaque materials. Therefore, consistent protocols are critical for making inter-study comparisons of micro-CT results, and analyses using standardized protocols should be performed to ensure the selection of the best-indicated dental material for a given purpose.

\section{Acknowledgments}

The authors wish to thank Renato Luiz Carvalho for his help in illustrating this paper.

\section{Conflicts of Interest: None}

\section{References}

1. Mao T, Neelakantan P. Three-dimensional imaging modalities in endodontics. Imaging Sci Dent 2014; 44: 177-83. 
2. Celikten B, Jacobs R, de Faria Vasconcelos K, Huang Y, Shaheen E, Nicolielo LF, et al. Comparative evaluation of cone beam CT and micro-CT on blooming artifacts in human teeth filled with bioceramic sealers. Clin Oral Investig 2019; 23: 3267-73.

3. Acar B, Kamburoğlu K, Tatar I, Arıkan V, Çelik HH, Yüksel S, et al. Comparison of micro-computerized tomography and conebeam computerized tomography in the detection of accessory canals in primary molars. Imaging Sci Dent 2015; 45: 205-11.

4. Tanomaru-Filho M, Torres FF, Chávez-Andrade GM, de Almeida M, Navarro LG, Steier L, et al. Physicochemical properties and volumetric change of silicone/bioactive glass and calcium silicate-based endodontic sealers. J Endod 2017; 43: 2097-101.

5. Marciano MA, Camilleri J, Costa RM, Matsumoto MA, Guimarães BM, Duarte MA. Zinc oxide inhibits dental discoloration caused by white mineral trioxide aggregate angelus. J Endod 2017; 43: 1001-7.

6. Basturk FB, Nekoofar MH, Gunday M, Dummer PM. Effect of various mixing and placement techniques on the flexural strength and porosity of mineral trioxide aggregate. J Endod 2014; 40: 441-5.

7. Viapiana R, Moinzadeh AT, Camilleri L, Wesselink PR, Tanomaru Filho M, Camilleri J. Porosity and sealing ability of root fillings with gutta-percha and BioRoot RCS or AH Plus sealers. Evaluation by three ex vivo methods. Int Endod J 2016; 49: 774-82.

8. Kang SW, Lee WJ, Choi SC, Lee SS, Heo MS, Huh KH, et al. Volumetric quantification of bone-implant contact using micro-computed tomography analysis based on region-based segmentation. Imaging Sci Dent 2015; 45: 7-13.

9. Rovaris K, Queiroz PM, Vasconcelos KF, Corpas LD, Silveira BM, Freitas DQ. Segmentation methods for micro CT images: a comparative study using human bone samples. Braz Dent $\mathbf{J}$ 2018; 29: 150-3.

10. Barrett WA, Mortensen EN. Interactive live-wire boundary extraction. Med Image Anal 1997; 1: 331-41.

11. Galibourg A, Dumoncel J, Telmon N, Calvet A, Michetti J, Maret D. Assessment of automatic segmentation of teeth using a watershed-based method. Dentomaxillofac Radiol 2018; 47: 20170220.

12. de Oliveira MV, Santos AC, Paulo G, Campos PS, Santos J. Application of a newly developed software program for image quality assessment in cone-beam computed tomography. Imaging Sci Dent 2017; 47: 75-86.

13. Bouxsein ML, Boyd SK, Christiansen BA, Guldberg RE, Jepsen KJ, Muller R. Guidelines for assessment of bone microstructure in rodents using micro-computed tomography. J Bone Miner Res 2010; 25: 1468-86.

14. Michetti J, Georgelin-Gurgel M, Mallet JP, Diemer F, Boulanouar K. Influence of CBCT parameters on the output of an automatic edge-detection-based endodontic segmentation. Dentomaxillofac Radiol 2015; 44: 20140413.

15. Huybrechts B, Bud M, Bergmans L, Lambrechts P, Jacobs R. Void detection in root fillings using intraoral analogue, intraoral digital and cone beam CT images. Int Endod J 2009; 42: 67585 .

16. Cooper D, Turinsky A, Sensen C, Hallgrimsson B. Effect of voxel size on 3D micro-CT analysis of cortical bone porosity.
Calcif Tissue Int 2007; 80: 211-9.

17. Kline TL, Knudsen BE, Anderson JL, Vercnocke AJ, Jorgensen SM, Ritman EL. Anatomy of hepatic arteriolo-portal venular shunts evaluated by 3D micro-CT imaging. J Anat 2014; 224 : 724-31.

18. Yamamoto-Silva FP, de Oliveira Siqueira CF, Silva MA, Fonseca RB, Santos AA, Estrela C, et al. Influence of voxel size on cone-beam computed tomography-based detection of vertical root fractures in the presence of intracanal metallic posts. Imaging Sci Dent 2018; 48: 177-84.

19. Jung M, Lommel D, Klimek J. The imaging of root canal obturation using micro-CT. Int Endod J 2005; 38: 617-26.

20. Inarejos Clemente EJ, Tolend M, Junhasavasdikul T, Stimec J, Tzaribachev N, Koos B, et al. Qualitative and semi-quantitative assessment of temporomandibular joint MRI protocols for juvenile idiopathic arthritis at 1.5 and 3.0T. Eur J Radiol 2018; 98 : 90-9.

21. Waarsing JH, Day JS, Weinans H. An improved segmentation method for in vivo microCT imaging. J Bone Miner Res 2004; 19: $1640-50$.

22. Spina TV, de Miranda PA, Falcão AX. Hybrid approaches for interactive image segmentation using the live markers paradigm. IEEE Trans Image Process 2014; 23: 5756-69.

23. Heckel F, Konrad O, Karl Hahn H, Peitgen HO. Interactive 3D medical image segmentation with energy-minimizing implicit functions. Comput Graph 2011; 35: 275-87.

24. EzEldeen M, Van Gorp G, Van Dessel J, Vandermeulen D, Jacobs R. 3-dimensional analysis of regenerative endodontic treatment outcome. J Endod 2015; 41: 317-24.

25. Zamparini F, Siboni F, Prati C, Taddei P, Gandolfi MG. Properties of calcium silicate-monobasic calcium phosphate materials for endodontics containing tantalum pentoxide and zirconium oxide. Clin Oral Investig 2019; 23: 445-57.

26. Bauer JS, Sidorenko I, Mueller D, Baum T, Issever AS, Eckstein F, et al. Prediction of bone strength by $\mu \mathrm{CT}$ and MDCTbased finite-element-models: how much spatial resolution is needed? Eur J Radiol 2014; 83: e36-42.

27. Lucas CP, Viapiana R, Bosso-Martelo R, Guerreiro-Tanomaru JM, Camilleri J, Tanomaru-Filho M. Physicochemical properties and dentin bond strength of a tricalcium silicate-based retrograde material. Braz Dent J 2017; 28: 51-6.

28. Choi JC, Choi CA, Yeo IL. Spiral scanning imaging and quantitative calculation of the 3-dimensional screw-shaped bone-implant interface on micro-computed tomography. J Periodontal Implant Sci 2018; 48: 202-12.

29. Marinozzi F, Bini F, Marinozzi A, Zuppante F, De Paolis A, Pecci R, et al. Technique for bone volume measurement from human femur head samples by classification of micro-CT image histograms. Ann Ist Super Sanita 2013; 49: 300-5.

30. Chen H, van Eijnatten M, Wolff J, de Lange J, van der Stelt PF, Lobbezoo F, et al. Reliability and accuracy of three imaging software packages used for 3D analysis of the upper airway on cone beam computed tomography images. Dentomaxillofac Radiol 2017; 46: 20170043.

31. Queiroz PM, Rovaris K, Gaêta-Araujo H, Marzola de Souza Bueno S, Freitas DQ, Groppo FC, et al. Influence of artifact reduction tools in micro-computed tomography images for endodontic research. J Endod 2017; 43: 2108-11. 
32. Sumitani Y, Hamba H, Nakamura K, Sadr A, Nikaido T, Tagami J. Micro-CT assessment of comparative radiopacity of adhesive/composite materials in a cylindrical cavity. Dent Mater J 2018; 37: 634-41.

33. Kim JE, Yi WJ, Heo MS, Lee SS, Choi SC, Huh KH. Three-dimensional evaluation of human jaw bone microarchitecture: correlation between the microarchitectural parameters of cone beam computed tomography and micro-computer tomography. Oral Surg Oral Med Oral Pathol Oral Radiol 2015; 120: 76270 .

34. Kerckhofs G, Schrooten J, Van Cleynenbreugel T, Lomov SV, Wevers M. Validation of X-ray microfocus computed tomography as an imaging tool for porous structures. Rev Sci Instrum 2008; 79: 013711.

35. Huang Y, Celikten B, de Faria Vasconcelos K, Ferreira Pinheiro Nicolielo L, Lippiatt N, Buyuksungur A, et al. Micro-CT and nano-CT analysis of filling quality of three different endodontic sealers. Dentomaxillofac Radiol 2017; 46: 20170223.

36. Orhan K, Jacobs R, Celikten B, Huang Y, de Faria Vasconcelos $\mathrm{K}$, Nicolielo LF, et al. Evaluation of threshold values for root canal filling voids in micro-CT and nano-CT images. Scanning 2018; 2018: 9437569.
37. Parkinson IH, Badiei A, Fazzalari NL. Variation in segmentation of bone from micro-CT imaging: implications for quantitative morphometric analysis. Australas Phys Eng Sci Med 2008; 31: 160-4.

38. Dietrich CA, Scheidegger CE, Schreiner J, Comba JL, Nedel LP, Silva CT. Edge transformations for improving mesh quality of marching cubes. IEEE Trans Vis Comput Graph 2009; 15: $150-9$.

39. Foteinos PA, Chernikov AN, Chrisochoides NP. Guaranteed quality tetrahedral Delaunay meshing for medical images. Comput Geom 2014; 47: 539-62.

40. Luo W, Pingel T, Heo J, Howard A, Jung J. A progressive black top hat transformation algorithm for estimating valley volumes on Mars. Comput Geosci 2015; 75: 17-23.

41. Dalili Z, Taramsari M, Mousavi Mehr SZ, Salamat F. Diagnostic value of two modes of cone-beam computed tomography in evaluation of simulated external root resorption: an in vitro study. Imaging Sci Dent 2012; 42: 19-24.

42. Choi JW. Factors affecting modulation transfer function measurements in cone-beam computed tomographic images. Imaging Sci Dent 2019; 49: 131-7. 